\title{
Studies of Silicon Photomultipliers and Preamplifier for the Wide Field of View Cherenkov Telescope Array of LHAASO
}

\author{
B. Y. Bi ${ }^{\star a b}$, S. S. Zhang ${ }^{a}$, Z. Cao ${ }^{a}$, L. Q. Yin ${ }^{a b}$, C. Wang ${ }^{a}$, T. Montarulic ${ }^{c}$, D. della Volpe ${ }^{c}$, \\ $M$. Heller ${ }^{c}$ for the LHAASO collaboration \\ ${ }^{a}$ Key Laboratory of Particle Astrophysics, Institute of High Energy Physics, CAS, Beijing, China \\ ${ }^{b}$ University of Chinese Academy of Sciences, Beijing, China \\ ${ }^{c}$ University of Geneva, Geneva, Switzerland \\ E-mail: bibydihep.ac.cn
}

\begin{abstract}
The Wide Field of View Cherenkov Telescope Array (WFCTA), a main component of the Large High Altitude Air Shower Observatory (LHAASO), requires the dynamic range from 10 to 32000 photoelectrons (pes) and the stable gain for the photosensors. Silicon photomultipliers (SiPMs) are new type of photosensors with respect to photomultiplier tubes (PMTs), the performance of which is improved rapidly since 1990s. SiPM do not suffer any ageing even with strong light exposure and the SiPM-based cameras could operate with high moonlight and their duty cycle is much larger than that of PMT-based cameras. In this paper, the design of preamplifier for the WFCTA camera is described. The dynamic range of SiPMs is studied, especially the non-linearity while the number of photons is large. A function is derived to describe the relationship between the number of fired cells and the total number of cells in the SiPM. The linearity of SiPMs follows the function well and could be corrected by the function. We also compared the performance of the SiPM and PMT under long time duration light pulses up to $3 \mu \mathrm{s}$, in order to investigate the stability of the gain of SiPMs. Moreover, the additional nonlinearities due to the nonuniform light distribution and the misestimation of the gain are also evaluated.
\end{abstract}

35th International Cosmic Ray Conference - ICRC2017

10-20 July, 2017

Bexco, Busan, Korea

${ }^{*}$ Speaker. 


\section{Introduction}

The Large High Altitude Air Shower Observatory (LHAASO), located at Mt. Haizi in Sichuan Province, China, is a hybrid experiment designed for $\gamma$-ray astronomy and cosmic ray (CR) physics [U, []. The Wide Field of View Cherenkov Telescope Array (WFCTA), one of the three main components of LHAASO, will be operated in two main observation modes. The Cherenkov mode to measure the energy spectrum of CRs from $30 \mathrm{TeV}$ to $300 \mathrm{PeV}$, requires the dynamic range of photosensors from 10 pes to 32,000 pes and the fluorescence mode to measure the ultra-high energy cosmic rays from hundreds of $\mathrm{PeV}$ to a couple of $\mathrm{EeV}$, requires that the gain of the sensors is stable for long duration light pulse up to $3 \mu s$.

The Silicon Photomultiplier (SiPM) is a relatively new type of photon sensor consisting of an array of independent avalanche photodiodes (APDs), so-called cells, which are connected in parallel and operated in Geiger mode [3]. The typical gain of SiPMs is around $10^{6}$ while the operating voltage is less than $100 \mathrm{~V}$. Compared with the PMT, the SiPM has many advantages such as high gain at low operating voltage, no aging due to strong light exposure. The SiPMbased cameras can operate under high moonlight condition, the duty cycle of which could increase from $10 \%$ up to $30 \%$ with respect to PMT-based cameras. The First G-APD Cherenkov Telescope (FACT) has been exploring the usage of the SiPM technology on IACT [䧃]. The single mirror Small Size Telescopes (SST-1M) and dual mirror SSTs of the Cherenkov Telescope Array (CTA) project will use SiPM [ [6].

The APDs in SiPMs are operated in Geiger mode, which means no matter how many photons hitting on one APD at the same time, the output of the APD will be the same. The fact causes that the dynamic range of SiPM is depended on the total number of cells and the uniformity of the distribution of photons hitting on the SiPM. In this paper, the SiPM samples and the design of the preamplifier for SiPM of WFCTA camera are described in Section 凹, as well as the test system and methods to study the properties of SiPMs. The results of experiments are shown in Section B, including dynamic range and performance under long duration light pulses of SiPMs. Additional nonlinearity due to non-uniformity of the distribution is simulated in this paper, and the details are discussed in Section 田.

\section{Experiment and Methods}

\subsection{The SiPMs and the Preamplifier}

We measured some samples from Hamamatsu, FBK and SensL and more details are listed in Table I. The dimensions of the avalanche photodiodes (APDs) are $25 \mu \mathrm{m}$ or $50 \mu \mathrm{m}$. The temperature coefficients are $54 \mathrm{mV} /{ }^{\circ} \mathrm{C}, 26 \mathrm{mV} /{ }^{\circ} \mathrm{C}$ and $21.5 \mathrm{mV} /{ }^{\circ} \mathrm{C}$ for samples from Hamamatsu, FBK and SensL respectively.

Fig. [(a) shows the design of preamplifier for LHAASO-WFCTA camera. The resistor $R_{2}$ converts the output of the SiPM from current to voltage, and influences the shape of the output signal. The pulses for different value of $R_{2}$ are shown in Fig. [(b). While the resistance $R_{2}=3 \Omega$, the FWHM of pulse is about $50 \mathrm{~ns}$, which is suitable for the $50 \mathrm{MHz}$ FADC in WFCTA electronics system. The preamplifier OPA846 is selected because of its high gain bandwidth and low input 
Table 1: Detail information of the SiPMs we studied, where H for Hamamatsu, F for FBK and S for SensL.

\begin{tabular}{cccccc}
\hline Models & PDE@ 400nm & Fill factor & Dark count rate & Cross talk & Gain $\left(10^{6}\right)$ \\
\hline S13361-3050AS-04(H) & $40 \%$ & $74 \%$ & $58 \mathrm{kHz} / \mathrm{mm}^{2}$ & $3 \%$ & 1.70 \\
S13361-5488 $(\mathrm{H})$ & $25 \%$ & $47 \%$ & $45 \mathrm{kHz} / \mathrm{mm}^{2}$ & $1 \%$ & 0.70 \\
FBK-25 $(\mathrm{F})$ & $38 \%$ & $72 \%$ & $80 \mathrm{kHz} / \mathrm{mm}^{2}$ & $15 \%$ & 1.38 \\
FBK-20 $(\mathrm{F})$ & $34 \%$ & $65 \%$ & $80 \mathrm{kHz} / \mathrm{mm}^{2}$ & $15 \%$ & 1.06 \\
MicroJ-30020 $(\mathrm{S})$ & $33 \%$ & $62 \%$ & $80 \mathrm{kHz} / \mathrm{mm}^{2}$ & $5 \%$ & 1.70 \\
\hline
\end{tabular}

noise. The capacitor $C_{1}$ is the bypass capacitor to keep the operating voltage of the SiPM stable, especially while there is a long light pulse.

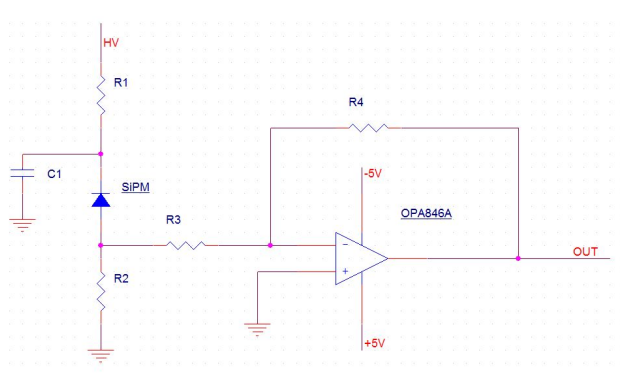

(a)

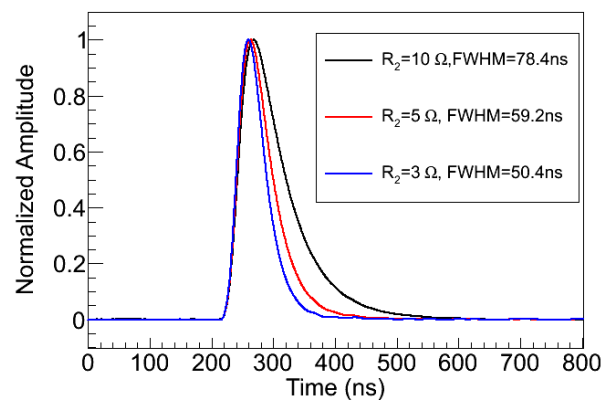

(b)

Figure 1: (a) The scheme of the preamplifier for the SiPM. (b) The pulses for different value of $R_{2}$ under a fixed intensity of light. The amplitudes are normalized to 1.

\subsection{Test Scheme and Platform}

The schematic diagram of the SiPM test system is shown in the Fig. 2(a). The one-dimensional sliding platform, the light source and the SiPM with the preamplifier are placed in a dark box, while the pulse generator and the oscilloscope are out. The temperature is kept at around $20{ }^{\circ} \mathrm{C}$ by air conditioner in the room. There are three temperature sensors placed very close to the SiPM to monitor the temperature to adapt the operating voltage on the SiPM, All these devices of the system are controlled by a computer, and the procedures are completed automatically.

As shown in Fig. 2(b), to measure the dynamic range of the SiPM, we set the LED at two fixed point: the near position $D_{1}$ and the far position $D_{2}$. The intensity of light at the two positions follow an inverse square law. The ratio of the intensity of light at the two positions is $R_{0}=8$ in our experiment. The brightness of the LED is kept unchanged, while the output of the SiPM at $D_{1}$ and $D_{2}$ is record. If the numbers of photoelectrons at the two position are $N_{p e}^{D_{1}}$ and $N_{p e}^{D_{2}}$ and the ratio is $R=N_{p e}^{D_{1}} / N_{p e}^{D_{2}}$, the relative deviation of the ratio from $R_{0}$ is defined as $D_{\text {line }}=\left(R-R_{0}\right) / R_{0} \times 100 \%$

The SiPM is operated in Geiger mode, thus saturation happens while more than one photons hit on the same APD in the same readout window. The response of the SiPM could be expressed as Eq. (‥ d) [G], and the number of photoelectrons could be derived from Eq. (‥J), and is expressed 


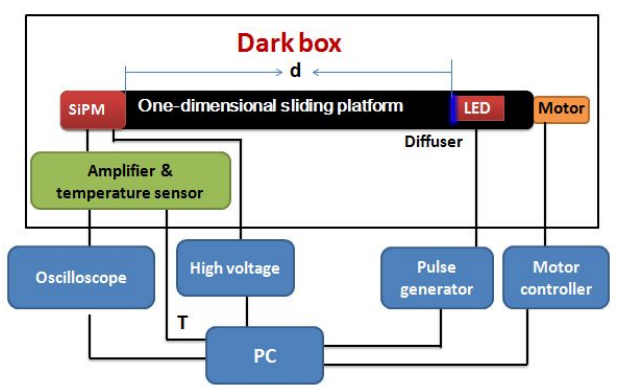

(a)

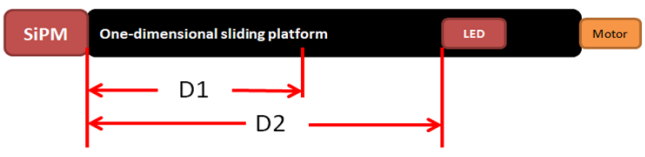

(b)

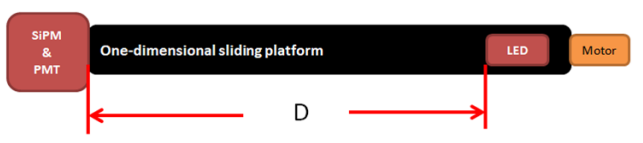

(c)

Figure 2: (a) The schematic diagram of the test platform. (b) The schematic diagram for the test of dynamic range. (c) The schematic diagram for test of performance under long duration light pulse.

as Eq. (2.2).

$$
\begin{aligned}
N_{\text {fired }} & =N_{\text {cell }}\left(1-e^{-\varepsilon N_{\text {ph }} / N_{\text {cell }}}\right)=N_{\text {cell }}\left(1-e^{-N_{\text {pe }} / N_{\text {cell }}}\right) \\
N_{p e}^{\text {rec }} & =N_{\text {cell }} \ln \left(\frac{1}{1-N_{\text {fired }} / N_{\text {cell }}}\right)
\end{aligned}
$$

where $N_{\text {fired }}$ is the number of fired APDs, which is the measurement from the SiPM. $N_{\text {cell }}$ is the total number of cells. $\varepsilon$ is the photon detection efficiency (PDE), $N_{p h}$ is the number of photons hitting on the SiPM, and then $\varepsilon N_{p h}$ is the number of photon electrons, so-called $N_{p e}$, which is the expected number of photoelectrons should be generated if there is no saturation due to the Geiger mode.

During a long time duration pulse, there is a current through the SiPM and the resistor $R_{1}$. The operating voltage on the SiPM will drop and the gain of the SiPM will be lower. The capacitor $C_{1}$ keeps the operating voltage as well as the gain of the SiPM stable, especially during a long light pulse.

In order to investigate the performance of the SiPM under long duration light pulses up to $3 \mu s$, we compared it with a R7899, which is the PMT satisfying the requirements of LHAASOWFCTA $[\square]$. A PMT is mounted close to the SiPM and the LED is moved far from the two devices. The time duration of the pulse is varied from $20 \mathrm{~ns}$ to $3 \mu \mathrm{s}$ while keeping the output of the PMT around $50 \mathrm{mV}$ to avoid the saturation of the SiPM, and record the output of the two devices. If $Q_{P M T}^{\Delta T}$ and $Q_{S i P M}^{\Delta T}$ are the output of the PMT and SiPM, respectively, while the duration time is $\Delta T$, and the ratio of the two outputs is $R_{\text {long }}^{\Delta T}=Q_{P M T}^{\Delta T} / Q_{S i P M}^{\Delta T}$, the stability of the gain could be indicated by the relative deviation of $R_{\text {long }}^{\Delta T}$ from $R_{\text {long }}^{20 n s}$, as $D_{\text {long }}=\left(R_{\text {long }}^{\Delta T}-R_{\text {long }}^{20 n s}\right) / R_{\text {long }}^{20 n s} \times 100 \%$

\section{Results}

Fig. 3(a) shows the dynamic ranges of all the SiPMs tested. The dynamic ranges are proportional to the total number of cells of the SiPM and follow the theoretical calculation with Eq. (D.d) very well. After correction by Eq. (I.2), the dynamic ranges of Hamamatsu and FBK samples are extended to more than 32000 pes. The dynamic range of SensL sample is about 6000 pes after correction because of its smaller $N_{\text {cell }}$. 


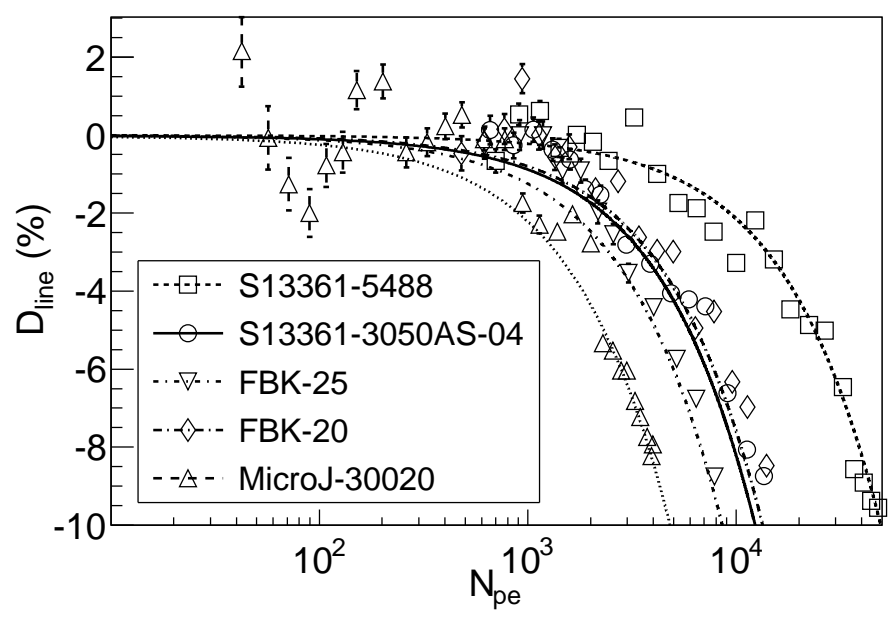

(a)

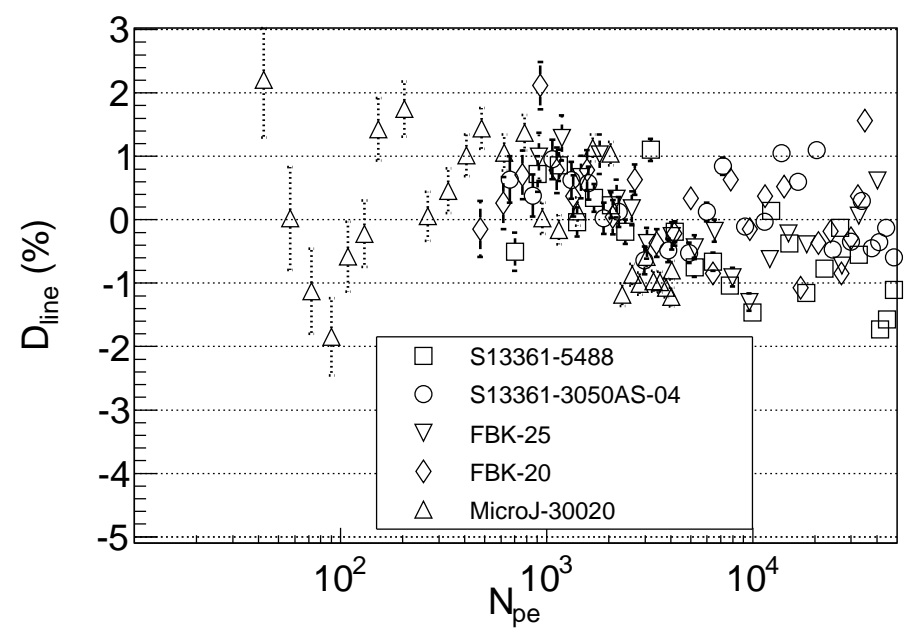

(b)

Figure 3: (a) Measurement of dynamic ranges of the samples from Hamamatsu, FBK and SensL. The lines in (a) are the expectation calculated by Eq. (‥ (ل). (b) Dynamic ranges after correction by Eq. (2.2). The nonlinearities of all samples are in the range of $\pm 2 \%$

The resolution is defined as $\sigma / N_{p e} \times 100 \%$ where $\sigma$ is the standard deviation of the distribution of $N_{p e}$. As shown in Fig. 4(a), the resolution of SiPMs are a little worse than a Poisson distribution which is the resolution of an ideal detector, due to noises from dark count, electronics and so on. The resolutions are almost the same before and after correction.

All of the telescopes will be operated as fluorescence telescopes, which requires the gain of the SiPM to be stable, especially under long duration pulses. According to the test result illustrated in Fig. 4(b), the value of $C_{1}$ influences the stability of the gain of the SiPMs while the duration of light getting longer. While $C_{1}=0.1 \mu F$ the performance of the SiPM is very poor, and while $C_{1}=1 \mu F$, the deviation of the gain of the SiPM is less than $\pm 2 \%$ from $20 \mathrm{~ns}$ to $3 \mu \mathrm{s}$. 


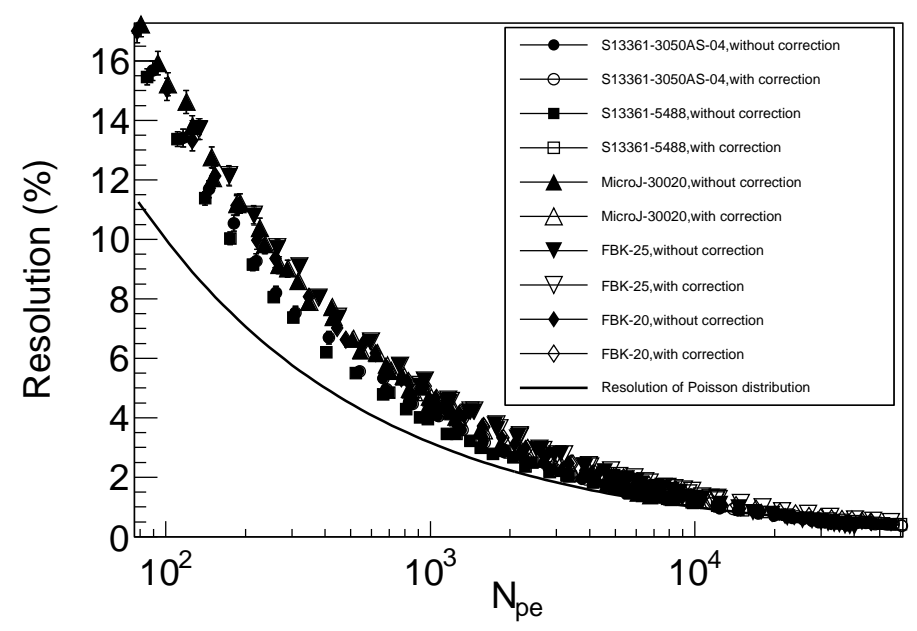

(a)

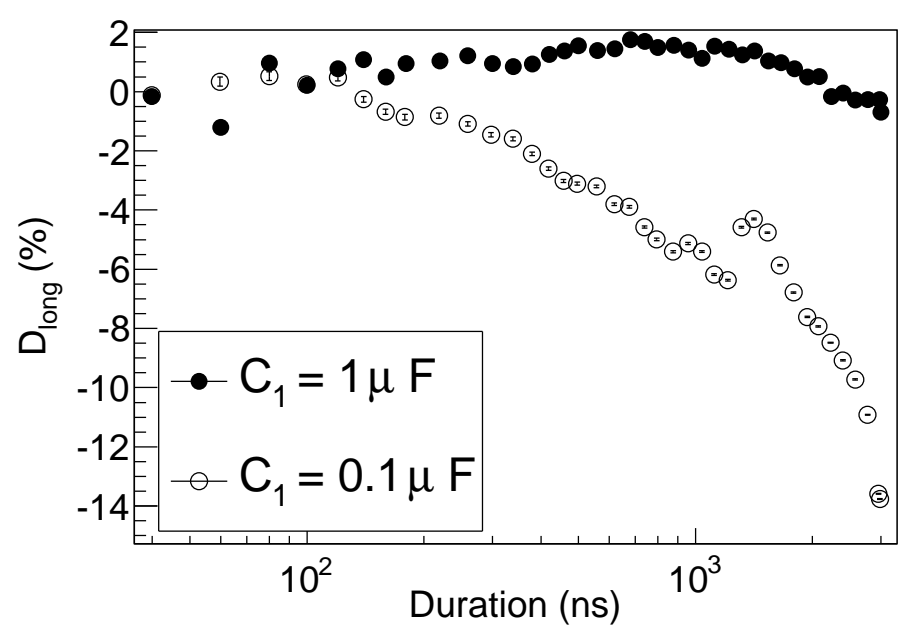

(b)

Figure 4: (a) Resolution of the SiPMs before and after correction by Eq. (2.2]). The correction does not influence the resolution. (b) Stability of the gain of S13361-3050AS-04 under long duration pulses up to $3 \mu \mathrm{s}$. The deviation of the gain is less than $\pm 2 \%$ when $C_{1}=1 \mu \mathrm{F}$.

\section{Additional Nonlinearities}

In the telescopes of WFCTA, photons are collected by sphere reflective mirrors and the camera is located at the focus plane of the mirrors, which makes the distribution is not uniform. The situation is further worsened by the light concentrators, so-called the Winston cones [ $[0]$, used to improve the photon collection efficiency. These two factors make the distribution of photons hitting on the SiPM non-uniform. Eq. (2.2) is based on the assumption that photons hit on the SiPM uniformly and the non-uniformity is only due to the two factors above. We investigated 
this situation by Monte-Carlo methods. The simulation was performed for primary proton with the energy range from $100 \mathrm{TeV}$ to $1 \mathrm{PeV}$, which is enough to study the saturation of the SiPM. All of the primary cosmic ray events, including Cherenkov photons, are generated by the program CORSIKA-v7.4005[Q], where the high energy hadronic interactions are simulated by QGSJETII04[ए]] and the low energy hadronic interactions by FLUKA(2011.2c)[प]]. A simulation program has been developed for LHAASO-WFCTA, including ray-tracing of photons, response of SiPM and the electronics, and also the effect of the light concentrators. The photons hitting on the SiPM are counted and the output of the SiPM is recorded. Fig. 5(a) shows the results of the simulation

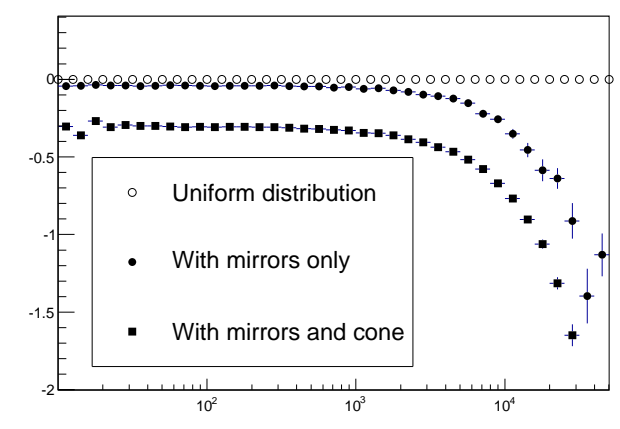

(a)

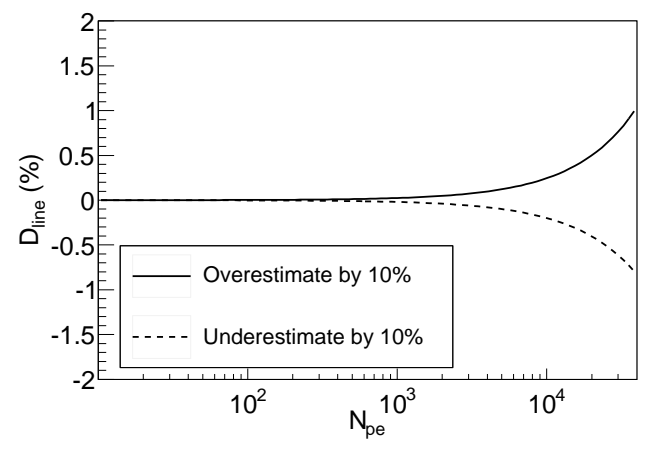

(b)

Figure 5: (a) Simulation of additional nonlinearity evaluated with Monte-Carlo program of WFCTA, including the mirrors and the concentrator. (b) Nonlinearity due to over/under estimation of the gain of the SiPM by $10 \%$.

for S13361-5488 from Hamamatsu. The total number of APDs of the SiPM is 230,400. If the distribution of light on the surface is uniform, the output of the SiPM is corrected perfectly by Eq. ([2.2), which is an ideal condition. In the real case, simulated with the Monte-Carlo program of WFCTA including the mirrors and the concentrators, the deviation is less than $2 \%$ after correction while the number of photoelectron is less than 32,000 pes.

The correction by Eq. ([2.2) requires an accurate calibration of the gain of the SiPM to get the correct value of $N_{\text {fired }}$. The nonlinearity due to the over/under estimation of the gain is easily calculated by Eq (R.d ) and ([2.2). Fig. 5(b) shows the result of the calculation. The deviation is less than $\pm 1 \%$ while overestimating or underestimating the gain by $10 \%$.

\section{Discussion and Conclusion}

The design of the preamplifier for the SiPM for LHAASO-WFCTA is illustrated in Fig. 1(a). The resistor chosen $R_{2}=3 \Omega$ makes sure the duration of the output signal suitable for sampling by ADC.

As shown in Fig. 3(a) the dynamic range is proportional to the total number of cells. The number of fired cells, the number of photoelectrons and the total number of cells are described by an exponential function (Eq. (D.J)) which could be used to correct the output number of photoelectrons. The dynamic range of SiPM follows well the theoretical line. The nonlinearity is less than $\pm 2 \%$ after correction by Eq. (‥2). 
All the telescopes will work in fluorescence mode, where signals can last from hundreds of nanoseconds to several microseconds. The bypass capacitor $C_{1}$ keeps the gain of the SiPM stable, especially under long duration pulse, as be chosen of $1 \mu F$ according to the result in Fig. 4(b)].

There is additional nonlinearity due to the uniform distribution of photons in real case with the mirrors and the concentrator. According to the simulation, while the total number of cells is 230,400, the dynamic ranges meet the requirements of LHAASO-WFCTA. We also calculate the additional non-linearity due to the over/under estimation of the gain of the SiPM, which is less than $\pm 1 \%$ while overestimating or underestimating the gain by $10 \%$. We have designed a new SiPM with 360,000 cells to make sure that there would not be saturation until more than 32,000 photoelectrons.

\section{Acknowledgements}

This work is supported in China by the Key Laboratory of Particle Astrophysics, Institute of High Energy Physics, CAS. Projects No. 11635011, No. 11475190 and No. 11675204 of NSFC also provide support to this study.

\section{References}

[1] Z. Cao, A future project at tibet: the large high altitude air shower observatory (LHAASO), Chinese Phys. C. 34 (2010) 249252.

[2] H. He, LHAASO Project : detector design and prototype, in: Proc. 31st ICRC, Lodz, Poland, 2009.

[3] N. Otte, The Silicon Photomultiplier-A new device for High Energy Physics, Astroparticle Physics, Industrial and Medical Applications, Proc. to SNIC Symp. (2006) 19.

[4] H. Anderhub, M. Backes, A. Biland, et al., Design and Operation of FACT - The First G-APD Cherenkov Telescope, J. Instrum. 8 (2013) P06008P06008.

[5] M. Heller, E. jr Schioppa, A. Porcelli, et al., An innovative silicon photomultiplier digitizing camera for gamma-ray astronomy, Eur. Phys. J. C. 77 (2017) 47

[6] H.T. van Dam, S. Seifert, R. Vinke, et al., A Comprehensive Model of the Response of Silicon Photomultipliers, IEEE Trans. Nucl. Sci. 57 (2010) 22542266.

[7] M. Ge, L. Zhang, Y. Chen, et al., Photomultiplier tube selection for the Wide Field of view Cherenkov/fluorescence Telescope Array of the Large High Altitude Air Shower Observatory, Nucl. Instruments Methods Phys. Res. Sect. A Accel. Spectrometers, Detect. Assoc. Equip. 819 (2016) 175181 .

[8] R. Winston, Light Collection within the Framework of Geometrical Optics, J. Opt. Soc. Am. 60 (1970) 245 .

[9] D. Heck, J. Knapp, J.N. Capdevielle, G. Schatz, T. Thouw, CORSIKA: A Monte Carlo Code to Simulate Extensive Air Showers, Forschungszentrum Karlsruhe. FZKA 6019 (1998) 190.

[10] S. Ostapchenko, Monte Carlo treatment of hadronic interactions in enhanced Pomeron scheme: QGSJET-II model, Phys. Rev. D. 83 (2011) 14018.

[11] A. Ferrari, P.R. Sala, A. Fasso, J. Ranft, FLUKA: A Multi-Particle Transport Code, (2005). 